\title{
Comparison of the safety of the application of painless gastroscopy and ordinary gastroscopy in chronic hypertension patients combined with early gastric cancer
}

\author{
FANGLI MEN $^{1 *}$, LIANMIN WEI $^{1 *}$, BING LIU $^{2}$, FENGTING WU ${ }^{1}$, JINGYU LIU $^{3}$, NI GUO $^{1}$ and QING NIU $^{1}$ \\ Departments of ${ }^{1}$ Gastroenterology, ${ }^{2}$ Tumor Radiotherapy and ${ }^{3}$ Anesthesiology, \\ Dongying People's Hospital, Dongying, Shandong 257091, P.R. China
}

Received August 18, 2017; Accepted November 30, 2017

DOI: $10.3892 / 01.2018 .7737$

\begin{abstract}
The aim of the present study was to compare the safety of the application of painless gastroscopy and ordinary gastroscopy for chronic hypertension patients combined with early gastric cancer. A total of 123 patients with early gastric cancer were selected at the Dongying People's Hospital from June, 2014 to August, 2016. The patients were randomly divided into the painless $(n=63)$ and ordinary $(n=60)$ gastroscopy groups. Proper pretreatment was performed according to whether anesthesia was performed or not. Arterial pressure, heart rate, and blood oxygen saturation were detected and compared before anesthesia, when gastroscope passed through the esophageal entrance plane, and after recovery from anesthesia. The incidence of nausea and vomiting, cough, dysphoria, throat discomfort and other adverse reactions during and after surgery were recorded and compared. Compared with the levels before anesthesia, the mean arterial pressure, heart rate and blood oxygen saturation were significantly reduced in painless gastroscopy when the gastroscope passed through the esophageal entrance plane $(\mathrm{P}<0.05)$. In the ordinary gastroscopy group, the mean arterial pressure, heart rate and blood oxygen saturation were significantly increased when the gastroscope passed through the esophageal entrance plane compared with the levels before anesthesia $(\mathrm{P}<0.05)$. Blood pressure decreased in the painless gastroscopy group whereas it increased in the ordinary gastroscopy group after anesthesia. The decrease in the painless gastroscopy group was lower than in the ordinary group. The incidence of intraoperative and postoperative adverse reactions including nausea,
\end{abstract}

Correspondence to: DrFangli Men, Department of Gastroenterology, Dongying People's Hospital, 317 East Chengnanyi Road, Dongying, Shandong 257091, P.R. China

E-mail: menfangli17@163.com

*Contributed equally

Key words: chronic hypertension, early gastric cancer, painless gastroscope, ordinary gastroscope vomiting, cough, dysphoria, pharyngeal discomfort and other adverse reactions was significantly decreased in the painless gastroscopy group than in the ordinary gastroscopy group $(\mathrm{P}<0.05)$. The results suggest that the application of painless gastroscopy in chronic hypertension patients can significantly reduce the incidence of intraoperative and postoperative adverse reactions compared with that of the Gastric cancer ordinary gastroscopy. Thus, painless gastroscopy is safer than ordinary gastroscopy.

\section{Introduction}

Approximately one million new cases of early gastric cancer are reported annually and account for $6.9 \%$ of all newly reported cancer cases (1). Gastric cancer ranks the fifth worldwide and is the third leading cause of cancer-related death after lung, breast, colorectal and prostate cancer $(1,2)$.

According to the depth of infiltration, gastric cancer is defined as limited infiltration to mucosa or submucosa, with or without lymphatic metastasis (3). Scholars in Japan and other countries consider that early detection and treatment is the most effective way to improve the prognosis of gastric cancer $(4,5)$. Although gastroscopy is safe for the general population, the application of gastroscopy in patients with chronic hypertension may cause arrhythmia, myocardial infarction and complications (6). Therefore, painless gastroscopy can be performed for patients with chronic hypertension under sedative conditions to reduce the pain and the incidence of restless situation.

The current study was carried out to compare the safety of the application of painless gastroscopy and ordinary gastroscopy in chronic hypertension patients combined with early gastric cancer.

\section{Materials and methods}

Materials. A total of 123 patients with suspected early gastric cancer were selected at the Dongying People's Hospital (Shandong, China) from June, 2014 to August, 2016. The current study was approved by the Ethics Committee of Dongying People's Hospital. Signed written informed consent was obtained from the patients. 
Table I. Comparison of general information of the two groups.

\begin{tabular}{|c|c|c|c|c|c|c|c|c|}
\hline \multirow[b]{2}{*}{ Groups } & \multirow[b]{2}{*}{$\mathrm{n}$} & \multirow[b]{2}{*}{$\begin{array}{l}\text { Age } \\
\text { years }\end{array}$} & \multicolumn{2}{|c|}{ Sex } & \multicolumn{3}{|c|}{ Symptoms (cases) } & \multirow[b]{2}{*}{$\begin{array}{c}\text { History of } \\
\text { hypertension (years }\end{array}$} \\
\hline & & & Male & Female & Anorexia & $\begin{array}{l}\text { Nausea and } \\
\text { vomitting }\end{array}$ & $\begin{array}{c}\text { Hematemesis } \\
\text { melena }\end{array}$ & \\
\hline $\begin{array}{l}\text { Painless } \\
\text { gastroscopy }\end{array}$ & 63 & $54.7 \pm 7.1$ & 30 & 33 & 33 & 29 & 1 & $11.3 \pm 6.1$ \\
\hline $\begin{array}{l}\text { Ordinary } \\
\text { gastroscopy }\end{array}$ & 60 & $52.7 \pm 6.8$ & 29 & 31 & 32 & 26 & 2 & $10.5 \pm 5.6$ \\
\hline$t / \chi^{2}$ & - & 8.72 & 10.11 & & 12.34 & 8.68 & 9.02 & 13.13 \\
\hline P-value & - & 0.09 & 0.39 & & 0.28 & 1.02 & 0.58 & 0.34 \\
\hline
\end{tabular}

Table II. Comparison of intraoperative and postoperative adverse reactions between the two groups [n (\%)].

\begin{tabular}{lccccc}
\hline Groups & Nausea & Vomiting & Throat discomfort & Cough & Dysphoria \\
\hline A & 0 & 0 & $3(4.8)$ & 0 & 0 \\
B & 60 & $43(71.7)$ & 60 & $48(80)$ & $25(41.7)$ \\
P-value & 0.047 & 0.038 & 0.026 & 0.034 & 0.049 \\
\hline
\end{tabular}

Patients were randomly divided into the painless gastroscopy $(n=63)$ and ordinary gastroscopy $(n=60)$ groups. Patients in the painless gastroscopy group included 30 males and 33 females, with an average age of of $54.7 \pm 7.1$ years. Patients in the ordinary group included 29 males and 31 females, with an average age of $52.7 \pm 6.8$ years. There was no significant difference in sex, age, early symptoms of gastric cancer and the history of hypertension between the two groups $(\mathrm{P}>0.05)$ (Table I).

General preparation. Preoperative preparation was performed for all the patients. Anesthesia and anesthesia-related complications were also explained to the patients. All the patients signed informed consent. Medical history was checked and clinical examination was performed to exclude disease of heart, brain and other vital organs. Patients were fasted for $8 \mathrm{~h}$ and deprivation was performed for $6 \mathrm{~h}$. Gastroscope, ECG monitor, mask, oxygen supplies and narcotic drugs were prepared.

Operation methods. Patients were fasted for $8 \mathrm{~h}$ and deprivation was performed for $4 \mathrm{~h}$ before treatment. Oral administration of dacronine hydrochloride (Yangtze River Pharmaceutical Group, Ltd., Jiangsu, China) was performed before gastroscopy for mucosal lubrication and anesthesia. Patients were fixed in left lateral position, and vital signs were checked. Patients in the painless gastroscopy were given balanced anesthesia before gastroscopy, and the specific method employed was: Remifentanil (Yichang Renfu Pharmaceutical Co., Ltd., Hubei, China) at a dose of $0.5-1 \mu \mathrm{g} / \mathrm{kg}$ and propofol (Beijing Fresenius Kabi Pharmaceutical Co., Ltd., Beijing, China) at a dose of $1.5-2 \mathrm{mg} / \mathrm{kg}$ through slow intravenous injection. Gastroscopy was performed when patients lost consciousness, relevant reflex disappeared, and muscle relaxed. Proper drug treatment was performed according to the conditions. When endoscope mirror body reached the descendant duodenum, images were captured and examination was stopped. Patients were asked not to intake any food and only intake liquid diet $2 \mathrm{~h}$ after operation. Balanced anesthesia was not performed for patients in the ordinary gastroscopy group.

Observation indicators. Changes in arterial pressure, heart rate, and blood oxygen saturation were recorded and compared before anesthesia, when the gastroscope passed through the esophageal entrance plane, and after recovery from anesthesia. The incidence of adverse reactions including nausea, vomiting, cough, dysphoria and throat discomfort were recorded and compared.

Statistical analysis. SPSS 11.0 statistical software (SPSS, Inc., Chicago, IL, USA) was used for the statistical analyses. Measurement data were expressed as mean $\pm \mathrm{SD}$, and comparisons between groups were performed using t-test. Countable data were compared using the $\chi^{2}$ test. $\mathrm{P}<0.05$ was considered to indicate a statistically significant analysis.

\section{Results}

Comparison of mean arterial pressure, heart rate and blood oxygen saturation between the two groups at different time points. Mean arterial pressure, heart rate and blood oxygen saturation were significantly reduced in painless gastroscopy when the gastroscope passed through the esophageal entrance plane compared with the levels of those factors before anesthesia $(\mathrm{P}<0.05)$. In the ordinary gastroscopy group, compared with the levels before anesthesia, the mean arterial pressure, heart rate and blood oxygen saturation were significantly increased when the gastroscope passed through the esophageal entrance plane $(\mathrm{P}<0.05)$. Blood pressure decreased in the painless gastroscopy group and increased in the ordinary 


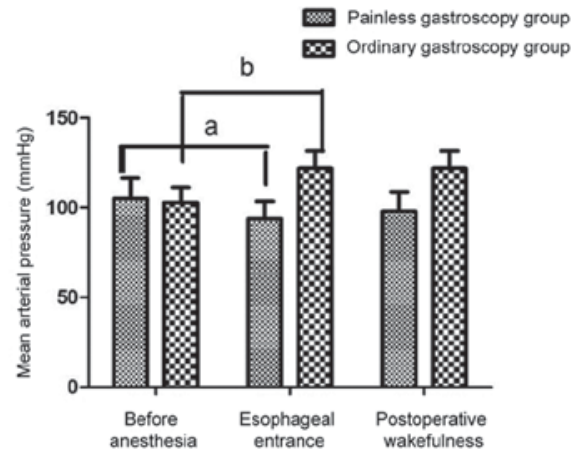

Figure 1. Comparison of mean arterial pressure between two groups. Mean arterial pressure was significantly reduced in the painless gastroscopy group when the gastroscope passed through the esophageal entrance plane compared with the levels of those factors before anesthesia ( $\left.{ }^{\mathrm{a}} \mathrm{P}<0.05\right)$. In the ordinary gastroscopy group, compared with the levels before anesthesia, mean arterial pressure was significantly increased when the gastroscope passed through the esophageal entrance plane ( $\left.{ }^{\mathrm{b}} \mathrm{P}<0.05\right)$.

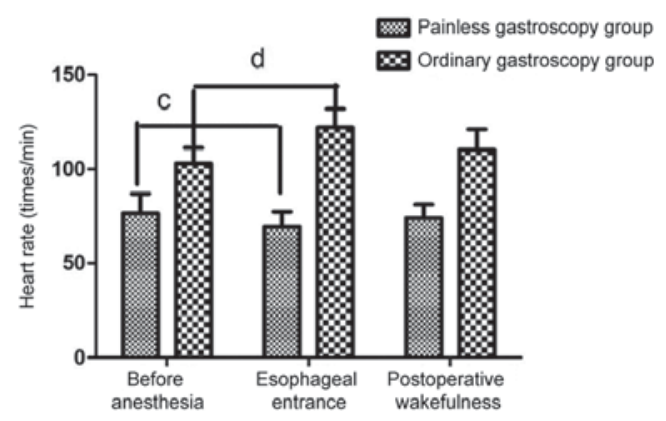

Figure 2. Comparison of heart rate between the two groups. Heart rate was significantly reduced in the painless gastroscopy group when the gastroscope passed through the esophageal entrance plane compared with the levels of those factors before anesthesia ( $\left.{ }^{\mathrm{C}} \mathrm{P}<0.05\right)$. In the ordinary gastroscopy group, compared with the levels before anesthesia, the heart rate was significantly increased when the gastroscope passed through the esophageal entrance plane $\left({ }^{\mathrm{d}} \mathrm{P}<0.05\right)$.

gastroscopy group after anesthesia. The decrease in the painless gastroscopy group was lower than the increase in the ordinary group (Figs. 1-4).

Comparison of intra- and postoperative adverse reactions between the two groups. The incidence of intra- and postoperative adverse reactions including nausea, vomiting, cough, dysphoria, throat discomfort and other adverse reactions was significantly lower in the painless gastroscopy group compared with the ordinary gastroscopy group $(\mathrm{P}<0.05)$. Thus, painless gastroscopy can reduce the incidence of postoperative adverse reactions (Table II).

Comparison of the degree of tolerance between the two groups. The number of patients showing no discomfort to gastroscopy was significantly smaller in the painless gastroscopy than in the ordinary gastroscopy $(\mathrm{P}<0.05)($ Table III).

\section{Discussion}

Employing gastroscopy is imperative in the diagnosis of gastric and upper gastrointestinal diseases, such as bleeding

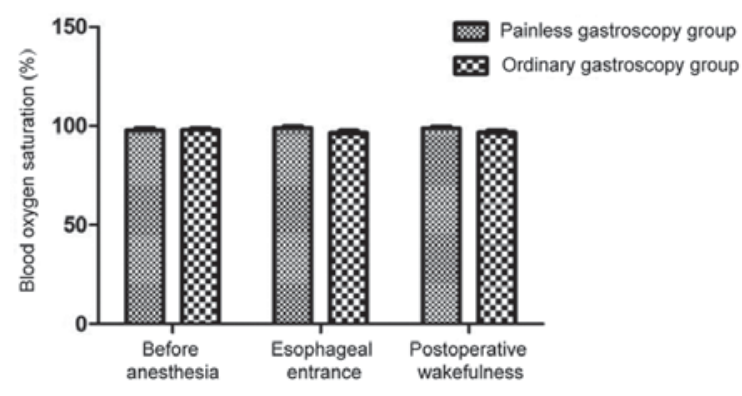

Figure 3. Comparison of blood oxygen saturation between the two groups No significant differences in blood oxygen saturation were found between the two groups.

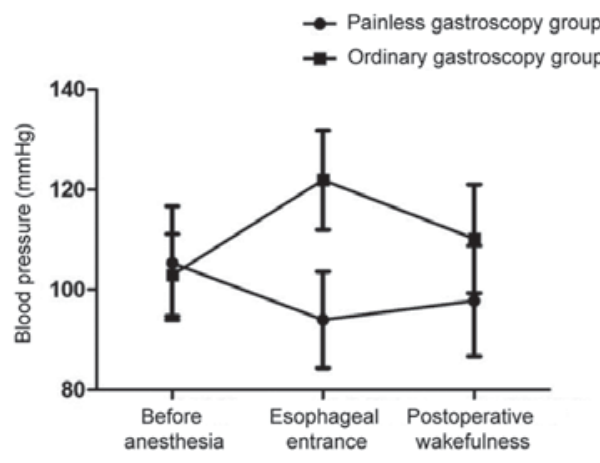

Figure 4. Comparison of blood pressure between the two groups. Blood pressure was reduced in the painless gastroscopy group and elevated in the ordinary gastroscopy group after anesthesia. The decrease in the painless gastroscopy group was lower than the increase in the ordinary group.

Table III. Comparison of degree of tolerance between the two groups [n (\%)].

\begin{tabular}{lccc}
\hline & & \multicolumn{2}{c}{ Discomfort } \\
\cline { 3 - 4 } Groups & Unbearable & Obviously & Minor \\
\hline $\begin{array}{l}\text { Painless } \\
\text { gastroscopy group }\end{array}$ & 0 & 0 & 2 \\
$\begin{array}{l}\text { Ordinary } \\
\text { gastroscopy group }\end{array}$ & $15(25)$ & $30(50)$ & $15 \mathrm{v} 25$ \\
\begin{tabular}{l} 
P-value \\
\hline
\end{tabular} & 0.030 & 0.042 & 0.021 \\
\hline
\end{tabular}

and ulcers. Due to its invasive nature, gastroscopy leads to pain in patients (7). Under ordinary gastroscopy, local irritation caused by the endoscope can induce nausea and vomiting. In addition, the effects of the hypothalamus-pituitary-adrenal cortical system lead to, a series of changes in vital signs of the body (8). Previous findings have shown that gastroscopy may cause a series of complications in patients with hypertension, such as myocardial infarction, cardiac arrest and other complications (9). The development of endoscopic technology, has led to an increase in the diagnostic rate of early gastric cancer. However, the incidence of hypertension is also on the increase. Therefore, the safe use of this technique has become a focus of current research. The use of painless gastroscopy, 
not only significantly reduces pain, but can also facilitate the diagnosis of gastric cancer.

The morbidity and mortality of gastric cancer are only lower to lung cancer $(10,11)$. Additionally, early diagnosis is closely related to the prognosis of patients (12). Propofol used in balanced anesthesia before painless gastroscopy can induce anesthesia rapidly, and the recovery is fast. Propofol can also inhibit nausea and vomiting. Nevertheless propofol has certain inhibitory effects on the respiratory and circulatory system, inducing a decrease in blood pressure and heart rate, albeit these effects are related to the dose administered (13). Remifentanil is a potent opioid receptor agonist with rapid action $(14,15)$. The combined use of propofol and remifentanil utilizes the advantages of both to maintain vital signs and reduce unnecessary adverse reactions, in order to facilitate gastroscopy (16-18).

Previous findings have shown that the incidence of digestive diseases is high in patients with chronic hypertension. In patients older than 45 years, the risk of cardiovascular disease can be increased by 2 -fold by an increase of $20 \mathrm{mmHg}$ in systolic blood pressure and $10 \mathrm{mmHg}$ in diastolic blood pressure $(19,20)$. In patients with chronic hypertension, blood pressure is altered during gastroscopy, which in turn increases the incidence of cerebrovascular disease. In the present study, an increase in blood pressure and heart rate was observed in the ordinary gastroscopy group, whereas, the drug used in the painless gastroscopy group reduced the blood pressure. Mean arterial pressure, heart rate and blood oxygen saturation were significantly reduced in painless gastroscopy when the gastroscope passed through the esophageal entrance plane compared with the levels of those factors before anesthesia $(\mathrm{P}<0.05)$. In the ordinary gastroscopy group, compared with the levels before anesthesia, mean arterial pressure, heart rate and blood oxygen saturation were significantly increased when the gastroscope passed through the esophageal entrance plane $(\mathrm{P}<0.05)$. No obvious discomfort, nausea, vomiting, cough, dysphoria, or pharyngeal discomfort was found in the painless gastroscopy group.

In conclusion, painless gastroscopy is safer, and more comfortable and effective for chronic hypertension patients combined with early gastric cancer. However, contraindications should be checked and vital signs should be monitored to reduce the intra- and postoperative bleeding caused by surgery. The application of painless gastroscopy can significantly increase the diagnostic rate of early gastric cancer. However, the circulation should be maintained to reduce complications of anesthesia.

\section{Competing interests}

The authors declare that they have no competing interests.

\section{References}

1. Jemal A, Bray F, Center MM, Ferlay J, Ward E and Forman D: Global cancer statistics. CA Cancer J Clin 61: 69-90, 2011.

2. Ferlay J, Soerjomataram I, Dikshit R, Eser S, Mathers C, Rebelo M, Parkin DM, Forman D and Bray F: Cancer incidence and mortality worldwide: Sources, methods and major patterns in GLOBOCAN 2012. Int J Cancer 136: 359-386, 2015.
3. Katai $\mathrm{H}$ and Sano T: Early gastric cancer: Concepts, diagnosis, and management. Int J Clin Oncol 10: 375-383, 2005.

4. Crew KD and Neugut AI: Epidemiology of gastric cancer. World J Gastroenterol 12: 354-362, 2006.

5. Tsubono $\mathrm{Y}$ and Hisamichi S: Screening for gastric cancer in Japan. Gastric Cancer 3: 9-18, 2000.

6. Yeoh KG: How do we improve outcomes for gastric cancer? J Gastroenterol Hepatol 22: 970-972, 2007.

7. Ross R and Newton JL: Heart rate and blood pressure changes during gastroscopy in healthy older subjects. Gerontology 50: 182-186, 2004

8. Maki S, Yao K, Nagahama T, Beppu T, Hisabe T, Takaki Y, Hirai F, Matsui T, Tanabe $\mathrm{H}$ and Iwashita A: Magnifying endoscopy with narrow-band imaging is useful in the differential diagnosis between low-grade adenoma and early cancer of superficial elevated gastric lesions. Gastric Cancer 16: 140-146, 2013.

9. Miskovitz P: Sleisenger \& Fordtran's gastrointestinal and liver disease-pathophysiology/diagnosis/management. Gastrointest Endosc 49: A2, 1999.

10. Takeno S, Hashimoto T, Maki K, Shibata R, Shiwaku H, Yamana I, Yamashita R and Yamashita Y: Gastric cancer arising from the remnant stomach after distal gastrectomy: A review. World J Gastroenterol 20: 13734-13740, 2014.

11. Fujiwara S, Yao K, Nagahama T, Uchita K, Kanemitsu T, Tsurumi K, Takatsu N, Hisabe T, Tanabe H, Iwashita A, et al: Can we accurately diagnose minute gastric cancers $(\leq 5 \mathrm{~mm})$ ? Chromoendoscopy (CE) vs magnifying endoscopy with narrow band imaging (M-NBI). Gastric Cancer 18: 590-596, 2015.

12. Tao G, Xing-Hua L, Ai-Ming Y, Wei-Xun Z, Fang Y, Xi W, Li-Yin W, Chong-Mei L, Gui-Jun F, Hui-Jun S, et al: Enhanced magnifying endoscopy for differential diagnosis of superficial gastric lesions identified with white-light endoscopy. Gastric Cancer 17: 122-129, 2014.

13. Disma N, Astuto M, Rizzo G, Rosano G, Naso P, Aprile G, Bonanno G and Russo A: Propofol sedation with fentanyl or midazolam during oesophagogastroduodenoscopy in children. Eur J Anaesthesiol 22: 848-852, 2005.

14. Dias-Silva D, Pimentel-Nunes P, Magalhaes J, Magalhaes R, Veloso N, Ferreira C, Figueiredo P, Moutinho P, and Dinis-Ribeiro M: The learning curve for narrow-band imaging in the diagnosis of precancerous gastric lesions by using web-based video. Gastrointest Endosc 79: 910-920, 2014.

15. Numata N, Oka S, Tanaka S, Kagemoto K, Sanomura Y, Yoshida S, Arihiro K, Shimamoto F and Chayama K: Risk factors and management of positive horizontal margin in early gastric cancer resected by en bloc endoscopic submucosal dissection. Gastric Cancer 18: 332-338, 2015.

16. Ichikawa D, Komatsu S, Kubota T, Okamoto K, Shiozaki A, Fujiwara $\mathrm{H}$ and Otsuji E: Long-term outcomes of patients who underwent limited proximal gastrectomy. Gastric Cancer 17: 141-145, 2014.

17. Ozcelik M, Guclu C, Bermede O, Baytas V, Altay N, Karahan MA, Erdogan B and Can O: The administration sequence of propofol and remifentanil does not affect the ED50 and ED95 of rocuronium in rapid sequence induction of anesthesia: A double-blind randomized controlled trial. Eur Rev Med Pharmacol Sci 20: 1479-1489, 2016.

18. Hwang SW and Lee DH: Is endoscopic ultrasonography still the modality of choice in preoperative staging of gastric cancer? World J Gastroenterol 20: 13775-13782, 2014.

19. Zhao P, Xiao SM, Tang LC, Ding Z, Zhou X and Chen XD: Proximal gastrectomy with jejunal interposition and TGRY anastomosis for proximal gastric cancer. World J Gastroenterol 20: 8268-8273, 2014

20. Amornyotin S, Lertakayamanee N, Wongyingsinn M, Pimukmanuskit $\mathrm{P}$ and Chalayonnavin V: The effectiveness of intravenous sedation in diagnostic upper gastrointestinal endoscopy. J Med Assoc Thai 90: 301-306, 2007.

This work is licensed under a Creative Commons Attribution-NonCommercial-NoDerivatives 4.0 International (CC BY-NC-ND 4.0) License. 